Original article

\title{
The positive effect of narcissism on depressive symptoms through mental toughness: Narcissism may be a dark trait but it does help with seeing the world less grey
}

\author{
Kostas A. Papageorgiou ${ }^{\mathrm{a}, \mathrm{b}, *}$, Andrew Denovan $^{\mathrm{c}}$, Neil Dagnall ${ }^{\mathrm{c}}$ \\ a School of Psychology, Queen's University Belfast, Malone Road, Belfast, United Kingdom \\ ${ }^{\mathrm{b}}$ Department of Psychology, Tomsk State University, Tomsk, Russia \\ ${ }^{\mathrm{c}}$ Department of Psychology, Manchester Metropolitan University, Manchester, United Kingdom
}

\section{A R T I C L E I N F O}

\section{Article history:}

Received 12 August 2018

Received in revised form 15 October 2018

Accepted 16 October 2018

Available online 1 November 2018

\section{Keywords:}

Grandiose narcissism

Mental toughness

Subclinical narcissism

Symptoms of depression

Vulnerable narcissism

\begin{abstract}
A B S T R A C T
Background: Subclinical Narcissism (SN) is part of the Dark Triad (DT), which includes also Subclinical Psychopathy (SP) and Machiavellianism. SN comprises facets retained from the clinical syndrome, such as grandiosity and dominance. Previous cross-sectional and longitudinal research indicates that SN may increase Mental Toughness (MT) resulting in various positive outcomes, including lower levels of psychopathy.

Method: The researchers conducted three studies ( $N=364,244$ and 144 for Study 1, 2 and 3 respectively) to test if the path model from SN to higher MT predicted lower symptoms of depression (DS). An extension to the model considered Openness to Experience (OE) as a possible mediator. Participants completed self-report measures of SN, MT, OE and DS. In Study 3, participants responded to an additional measure of $\mathrm{SN}$ to allow differentiation between grandiose and vulnerable aspects.

Results: SN exerted a negative indirect effect on DS through MT across studies; and a negative indirect effect on DS through MT and OE in Study 2. In Study 3, Grandiose SN increased MT contributing to lower DS. Vulnerable SN demonstrated the reverse pattern. MT subfactors of Control and Confidence had a mediating effect across studies.

Conclusion: The current findings support the model that SN to MT predicts positive outcomes in various domains, including lower levels of psychiatric symptoms. Exploring the link between SN with prosocial traits can be particularly helpful when seeking to identify and promote SN's adaptive tendencies against symptoms of psychopathology.
\end{abstract}

Crown Copyright @ 2018 Published by Elsevier Masson SAS. All rights reserved.

\section{Introduction}

Various personality factors are associated with symptoms of psychopathology [1]. Symptoms of psychopathology vary depending upon an individual's personality traits, and personality traits may influence the presence of symptoms of psychopathology [2]. Exploring factors that potentially guard against psychopathology, such as indicators of depression (DS) is of paramount importance because of the prevalence of depression (there are over 298 million sufferers worldwide) [3]. Depression has been associated with an increased risk of mortality in general community populations, as well as in patient populations with chronic illnesses, such as

\footnotetext{
* Corresponding author at: School of Psychology, Queen's University Belfast, David Keir Building, 18-30 Malone Road, Belfast, BT9 5BN, United Kingdom. E-mail address: K.Papageorgiou@qub.ac.uk (K.A. Papageorgiou).
}

coronary heart disease, cancer, diabetes and stroke [4]. The researchers conducted three studies to explore the degree to which Subclinical Narcissism (SN) predicted indirectly lower DS, through Mental Toughness (MT) and the Big Five (BF) personality trait of Openness to Experience (OE).

\section{Subclinical narcissism, mental toughness and psychopathology}

Subclinical Narcissism (SN) is part of the Dark Triad (DT), a personality cluster defined at the subclinical level. This includes the traits of Subclinical Psychopathy (SP) and Machiavellianism [5]. $\mathrm{SN}$ includes facets retained from the clinical syndrome, namely grandiosity, entitlement, dominance and superiority [5]. SN includes two main types: Grandiose Narcissism (GN) and Vulnerable Narcissism (VN) [6]. GN is characterised by exhibitionism, lack of humility/modesty and interpersonal dominance. VN 
encompasses negative affect, distrust, selfishness, and a need for attention and recognition [7,8]. Research suggests that GN links mainly to positive outcomes, whereas VN predicts negative outcomes [9]. For example, a recent study observed that participants scoring high on VN also reported higher DT traits, lower Mental Toughness (MT), poor sleep quality, and higher scores on perceived stress [10].

Previous studies suggest that SN might be unique among the DT traits in that, it encapsulates to a large extent prosocial and adaptive behaviours [11]. For example, a series of five studies indicated positive associations between $\mathrm{SN}$ and daily and dispositional subjective well-being, and couple well-being. Negative associations were found between SN and daily sadness, dispositional depression, daily and dispositional loneliness, daily anxiety and dispositional neuroticism [12].

More recently, several studies $[13,14,15,16]$ have reported moderate positive correlations between SN and MT. MT reflects an effective coping mechanism as reaction to stressors. Specifically, MT allows individuals to proactively seek out opportunities for personal growth [17]. Many studies have shown that MT is a personality trait that acts as a resilient factor against psychopathology (see [18] for a review). Congruently, both cross-sectional and longitudinal research has consistently linked high MT to significantly lower DS [19-21].

In terms of the factors that may influence MT, studies using both cross-sectional and longitudinal designs have shown that SN may increase MT. This results in various positive outcomes, such as lower psychopathy and higher school grades $[15,16]$. Furthermore, the authors concluded that the path model from SN to MT to a further outcome might predict positive effects across various contexts, including psychopathology.

\section{Subclinical narcissism, mental toughness and openness to experience}

Both SN and MT have been associated with the Big Five trait of Openness to Experience (OE) [22,23]. In fact, a study reported that the correlation between SN and OE was the strongest out of all correlations between SN and the other four BF traits (Conscientiousness, Extraversion, Neuroticism and Agreeableness) [22]. Another study reported that OE correlated with MT and in particular, with the MT facets of interpersonal confidence and challenge [23]. Confidence is the strongest correlate (out of all four facets of MT) between MT and SN [15]. The link between Challenge and $\mathrm{OE}$ may be explained conceptually by the fact that individuals that score high on Challenge perceive change and new experiences as an opportunity for growth rather than as a threat [24].

Collectively, these findings suggest that individuals scoring high on SN and MT may be particularly open to experiences. Particularly, that they possess the inclination and confidence to seek out new opportunities for personal growth.

\section{The present investigation}

The present investigation explored a statistical model testing the notion that SN increases MT and OE contributing indirectly to lower DS. Extensions to the model further examined whether the grandiose, as opposed to the vulnerable aspect of SN, increased MT leading to positive outcomes. It was hypothesised that [1] SN would exert a significant negative indirect effect on DS through MT across studies. The researchers posited also that [2] SN would exert a significant negative indirect effect on DS through MT and OE in Study 2 and 3. Furthermore, it was predicted [3] that GN would exert a significant negative indirect effect on DS through MT and OE (Study 3). Finally, it was anticipated [4] that VN would exert a significant positive indirect effect on DS through MT and OE (Study 3).

\section{Method}

\subsection{Sample}

Three studies tested hypotheses 1-4. The first examined whether SN exerted a significant negative indirect effect on DS through MT. The second replicated the findings of the first study with an independent sample, and developed the model by testing whether SN exerted a significant negative indirect effect on DS through MT and OE. The third replicated the findings of Studies 1 and 2, and further extended the model by assessing the differential effect of GN (as opposed to VN) on DS through MT and OE. Progressive extension of the tested model provided an increasingly sophisticated understanding of variable relationships.

\subsubsection{Study 1}

Participants $(N=364)$ were recruited online through advertisements on social networks (e.g., Facebook) and word of mouth. Participant mean age was 24.31 years $(S D=9.16$, range $=18-79)$; $56.9 \%$ were females. Inspection of univariate outliers identified that three z-scores exceeded 3.5 standard deviations (SDs). These were removed [25]. Multivariate outliers exist when data points exceed Cook's distance of 1.0 [26]. No responses surpassed Cook's distance. Skewness values were within the recommended range of -2.0 to +2.0 SDs from the mean [27]. Specifically, MT $=-0.32$; Challenge $=-0.27$; Commitment $=-0.14 ;$ Control $=-0.42$; Confidence $=-0.40 ;$ Narcissism $=-0.01 ;$ Depression $=1.33$. Participants did not receive compensation for taking part.

\subsubsection{Study 2}

Participants $(N=364)$ were also recruited online through advertisements on social networks (e.g., Facebook) and word of mouth. Participant mean age was 25.30 years $(S D=7.22$, range $=$ $18-59) ; 64.75 \%$ females. Examination of outliers resulted in the removal of five data points. No issues existed with skewness, $\mathrm{MT}=0.12$; Challenge $=-0.58$; Commitment $=-0.02$; ontrol $=0.15$;

Table 1

Descriptive statistics and correlations for Study 3 variables $(N=140)$.

\begin{tabular}{|c|c|c|c|c|c|c|c|c|c|c|c|c|c|}
\hline Variable & $M$ & $S D$ & Skew & 1 & 2 & 3 & 4 & 5 & 6 & 7 & 8 & 9 & 10 \\
\hline 1. Grandiosity & 2.38 & 0.51 & 0.70 & & -0.07 & $0.70^{* * *}$ & $0.40^{* * *}$ & $0.37^{* * *}$ & $0.21^{*}$ & $0.31^{* * *}$ & $0.45^{* * *}$ & 0.11 & $-0.19^{*}$ \\
\hline 2. Vulnerability & 3.13 & 0.63 & -0.36 & & & $-0.17^{*}$ & $-0.66^{* * *}$ & $-0.57^{* * *}$ & $-0.48^{* * *}$ & $-0.67^{* * *}$ & $-0.62^{* * *}$ & -0.05 & $0.53^{* * *}$ \\
\hline 3. Narcissism & 2.56 & 0.57 & 0.25 & & & & $0.46^{* * *}$ & $0.37^{* * *}$ & $0.29^{* *}$ & $0.31^{* * *}$ & $0.51^{* * *}$ & $0.23^{* *}$ & $-0.21^{*}$ \\
\hline 4. Mental Toughness & 3.25 & 0.42 & -0.32 & & & & & $0.77^{* * *}$ & $0.84^{* * *}$ & $0.85^{* * *}$ & $0.89^{* * *}$ & $0.22^{* *}$ & $-0.64^{* * *}$ \\
\hline 5. Challenge & 3.38 & 0.56 & 0.01 & & & & & & $0.57^{* * *}$ & $0.62^{* * *}$ & $0.64^{* * *}$ & $0.31^{* * *}$ & $-0.43^{* * *}$ \\
\hline 6. Commitment & 3.35 & 0.56 & -0.16 & & & & & & & $0.66^{* * *}$ & $0.60^{* * *}$ & $0.23^{* *}$ & $-0.51^{* * *}$ \\
\hline 7. Control & 3.02 & 0.47 & -0.31 & & & & & & & & $0.68^{* * *}$ & 0.11 & $-0.62^{* * *}$ \\
\hline 8. Confidence & 3.19 & 0.57 & -0.33 & & & & & & & & & 0.13 & $-0.57^{* * *}$ \\
\hline 9. Openness & 3.51 & 0.60 & -0.08 & & & & & & & & & & 0.01 \\
\hline 10. Depression & 10.34 & 8.16 & 1.16 & & & & & & & & & & \\
\hline
\end{tabular}

Note. ${ }^{*} p<0.05,{ }^{* *} p<0.01,{ }^{* * *} p<0.001$. 
Confidence $=-0.12 ;$ Narcissism $=-0.09 ;$ Openness $=-0.20$; Depression $=1.19$. Participants received no compensation for taking part.

\subsubsection{Study 3}

Participants $(N=144)$ were undergraduate students recruited through advertisements in the class and university. They enrolled to participate through the Sona system, which manages research participation. Specifically, it allows students to browse ongoing research and enrol to studies. Participant mean age was 22.08 years $(S D=5.50$, range $=18-52) ; 86.1 \%$ females. Twenty-three participants did not disclose their age. Assessment of univariate outliers supported removal of four data points. Cook's distance values were below 1.0. Skewness results fell between -2 and +2 SDs (Table 1). Once participants completed the study, they received credits as part of course fulfilment.

\subsection{Measures}

\subsubsection{Study 1}

Used the 9-item narcissism scale of the Short Dark Triad questionnaire (SD3) [28] to assess Subclinical Narcissism (Cronbach's alpha $=0.70$ ). The Mental Toughness Questionnaire 48 (MTQ48) [24] measured the four dimensions of MT (Control, Confidence, Challenge and Commitment, respectively) and total MT (Cronbach's alpha $=0.88,0.53,0.80,0.53$, and 0.80 for total MT). The Patient Health Questionnaire 9 (PHQ-9) [31] assessed the DSM-IV symptoms of major depressive disorder (Cronbach's alpha $=0.76)$.

\subsubsection{Study 2}

Again, employed the SD3 [28] (Cronbach's alpha = 0.67), MTQ48 [24] (Cronbach's alpha $=0.88,0.50,0.81,0.58$, and 0.74 for total MT), and the PHQ-9 [31] (Cronbach's alpha =0.86). These were presented alongside the 10-item scale of the Big Five Inventory (BFI) [30], which measured OE (Cronbach's alpha =0.72).

\subsubsection{Study 3}

Once more used the SD3 [28] (Cronbach's alpha = 0.73), MTQ48 [24] (Cronbach's alpha $=0.92,0.72,0.84,0.74$, and 0.82 for total MT) and the 10-item BFI [30] (Cronbach's alpha=0.76). These core scales were presented together with the Five-Factor Narcissism Inventory - Short Form (FFNI-SF) [29] (Cronbach's alpha $=0.92$ and 0.82 for the scales of Grandiose and Vulnerable Narcissism), and The Beck Depression Inventory (BDI) [32] (Cronbach's alpha = 0.90 ). Detailed information on the measures appears in the supplementary material section.

\subsection{Procedure}

Combined measures formed a single document. In Study 1 and 2, the online platform SurveyMonkey (www.surveymonkey.com) hosted this in electronic form. Participants accessed the questionnaire via a message containing a link, a password and a unique participant code. For Study 3, participants completed paper and pencil copies of the booklets within the university. Questionnaire completion was self-paced.

\subsection{Analysis}

Inspection of descriptive statistics and intercorrelation preceded tests of mediation. Mediation analysis used Process (Model 4) [42] with bootstrapping (1000 resamples) to generate indirect effect estimates with 95\% bias-corrected confidence intervals.

In Study 1, the mediation model examined DS as the outcome variable, SN as the predictor and MT as a mediator. Study 2 assessed
$\mathrm{OE}$ in addition to MT as mediators of the SN-DS relationship. Mediation analysis in Study 3 built upon Study 2 by testing whether facets of SN (GN and VN) had an indirect effect on DS through MT and OE. Specifically, Model 3 assessed GN as a predictor (controlling for VN). Mediator variables were MT and OE, with DS the outcome. Analysis of Model 4 tested VN as a predictor (controlling for GN). For comparison purposes, analysis considered also total SN in Model 5. Given MT is multidimensional [34], subfactors of Challenge, Commitment, Control and Confidence were examined as mediators in each study in addition to total MT.

For assessing mediation, various effects and statistical weights exist. The total effect ( $c$ weight) of a predictor on an outcome comprises an indirect effect $\left(a^{*} b\right.$ weight) and a direct effect controlling for the influence of a mediator ( $c$ ' weight). Weight $a$ relates to the effect of the predictor on the mediator. Weight $b$ is the effect of the mediator on the outcome while excluding the effect of the predictor. An indirect effect represents a combination of the regression weight of the predictor on the mediator and the regression weight of the mediator on the outcome.

\section{Results}

\subsection{Study 1}

Inspection of descriptive statistics and intercorrelations revealed significant relationships between $\mathrm{SN}(M=2.93, S D=0.57)$ and MT $(M=3.44, S D=0.37)(r=0.40)$, SN and DS $(M=5.43$, $S D=4.52)(r=-0.16)$, and MT and DS $(r=-0.53)$. Analysis of Model 1 (Fig. 1) found that SN positively predicted MT and negatively predicted DS ( $a$ weight). MT possessed a negative relationship with DS ( $b$ weight) and a mediating effect as indicated by a significant $a^{*} b$ weight. The direct effect of SN was non-significant ( $c$ ' weight), supporting presence of mediation in addition to a meaningful indirect effect. Since the model was cross-sectional, reversing the paths between DS and SN revealed a considerably weaker indirect effect ( $a^{*} b$ weight $=-0.02$ vs. -1.77 ). Similarly, a model assessing $\mathrm{SN}$ as mediator between MT-DS revealed a non-significant indirect effect, $a^{*} b=0.31,95 \% \mathrm{CI}[-0.16,0.84]$, supporting the direction in Model 1.

Further scrutiny of MT subfactors revealed significant indirect effects of SN on DS through Challenge, $a^{*} b=0.40,95 \% \mathrm{CI}[0.18,0.74]$, Commitment, $a^{*} b=-0.29$, 95\% CI $[-0.64,-0.08]$, Control, $a^{*} b=$ $-0.32,95 \% \mathrm{CI}[-0.63,-0.11]$, and Confidence, $a^{*} b=-1.86,95 \% \mathrm{CI}$ $[-2.60,-1.22]$. All subfactors were in the expected direction apart from Challenge, which evidenced a positive mediating effect on DS. Study 1 data available via figshare: https://doi.org/10.6084/m9. figshare.6887123.

\subsection{Study 2}

Analysis followed the same steps as Study 1. Descriptive statistics and intercorrelations revealed significant relationships between SN $(M=2.88, S D=0.55)$ and MT $(M=3.42, S D=0.38)$

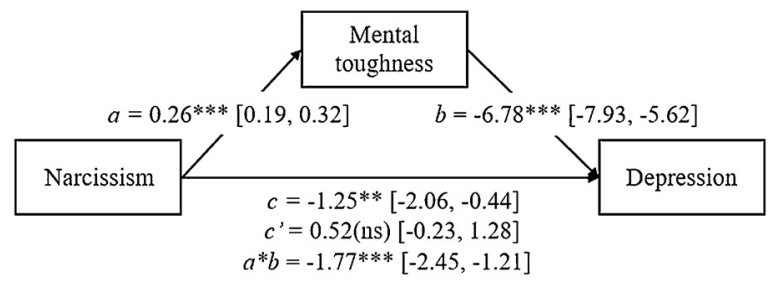

Fig. 1. Model 1: Mediation effects of Mental Toughness in the relation between narcissism and depression. Note. $\mathrm{R}^{2}$ for final model $=28.89 \%$; results are based on 1000 bootstrap samples; values in square brackets indicate bias-corrected $95 \%$ confidence intervals; ${ }^{* *} p<0.01,{ }^{* * *} p<0.001$. 
$(r=0.44)$, SN and $\mathrm{OE}(M=3.46, S D=0.51)(r=0.27)$, MT and DS $(M=$ $7.13, S D=5.43)(r=-0.51)$, and $S N$ and DS $(r=-0.13)$. No significant association existed between $\mathrm{OE}$ and DS.

Analysis of Model 2 (Fig. 2) indicated that SN positively predicted MT and OE ( $a$ weights). MT negatively predicted DS, whereas OE positively predicted DS ( $b$ weights). SN had a significant indirect effect on DS through MT and OE ( $a^{*} b$ weights). Additionally, a non-significant $c$ ' path supported mediation. Reversing paths between SN and DS revealed considerably weaker indirect effects (MT $a^{*} b$ weight $=-0.02$ vs. -2.59 ; OE $a^{*} b$ weight $=0.01$ vs. 0.52). Assessing SN as mediator of MT-DS revealed a non-significant indirect effect, $a^{*} b=0.43,95 \% \mathrm{CI}$ $[-0.24,1.14]$. Testing MT as a mediator of OE-DS (controlling for $\mathrm{SN}$ ) reported a non-significant total effect, $c=0.05,95 \% \mathrm{CI}[-0.08$, $0.19]$, supporting variable direction in Model 2. OE possessed a non-significant correlation with DS but a significant mediating effect. Possibly, this was due to shared variance with SN and MT. The correlation between $\mathrm{OE}$ and DS reached significance when controlling for the effects of SN and MT, partial $r=0.22, p=0.001$.

Scrutiny of MT subfactors revealed significant indirect effects of SN on DS via Control, $a^{*} b=-3.48,95 \% \mathrm{CI}[-5.86,-1.11]$, and Confidence, $a^{*} b=-4.24,95 \% \mathrm{CI}[-5.98,-2.51]$. As with Model 2, OE exerted a significant mediating effect, $a^{*} b=0.41,95 \% \mathrm{CI}[0.15,0.80]$. This is likely due to shared variance given the correlation between OE and DS was significant when controlling for SN and MT subfactors, partial $r=0.17, p=0.008$. Study 2 data available through figshare: https://doi.org/10.6084/m9.figshare.6887132.

\subsection{Study 3}

Consideration of zero-order correlations revealed several significant associations (Table 1). Grandiose Narcissism (GN), SN and MT correlated positively. OE was positively associated with SN and MT. DS correlated negatively with GN, SN and MT, and positively with Vulnerable Narcissism (VN). There was no significant relationship between DS and $\mathrm{OE}$.

Model 3 (Fig. 3) revealed GN positively predicted MT (not OE) and VN negatively predicted MT (not OE) ( $a$ weights). MT and OE revealed negative and positive relationships with DS respectively. GN had a significant indirect effect on DS through MT ( $a^{*} b$ weight). OE did not have a mediating effect.

Model 4 (Fig. 3 ) reported identical $a$ and $b$ weights. VN had a significant indirect effect on DS via MT ( $a^{*} b$ weight). OE did not have a mediating effect. Note the negative relationship between MT and DS in Model 3 and 4, and the non-significant direct effects of GN and VN (c' weights). These results, alongside significant indirect effects, support mediation. Reversing relationships reported a comparatively weaker indirect effect through MT with both GN ( $a^{*} b$ weight $=-0.01$ vs. -3.26$)$ and $\mathrm{VN}\left(a^{*} b\right.$ weight $=0.02$ vs. 4.66). Similarly, testing MT as a mediator of OE-DS (controlling

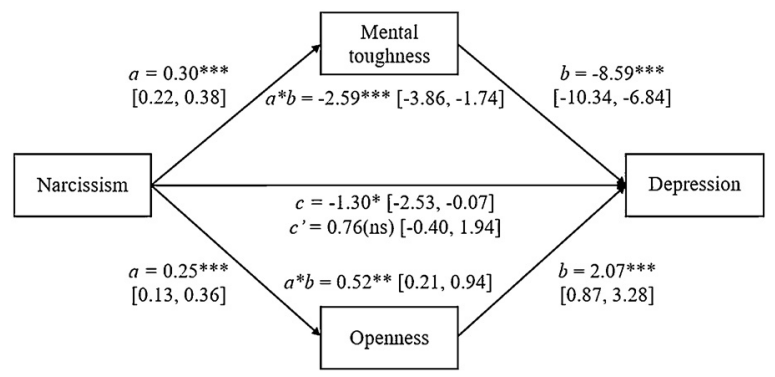

Fig. 2. Model 2: Mediation effects of Mental Toughness and openness in the relation between narcissism and depression. Note. $\mathrm{R}^{2}$ for final model $=30.03 \%$; results are based on 1000 bootstrap samples; values in square brackets indicate bias-corrected $95 \%$ confidence intervals; ${ }^{*} p<0.05,{ }^{* *} p<0.01,{ }^{* * *} p<0.001$
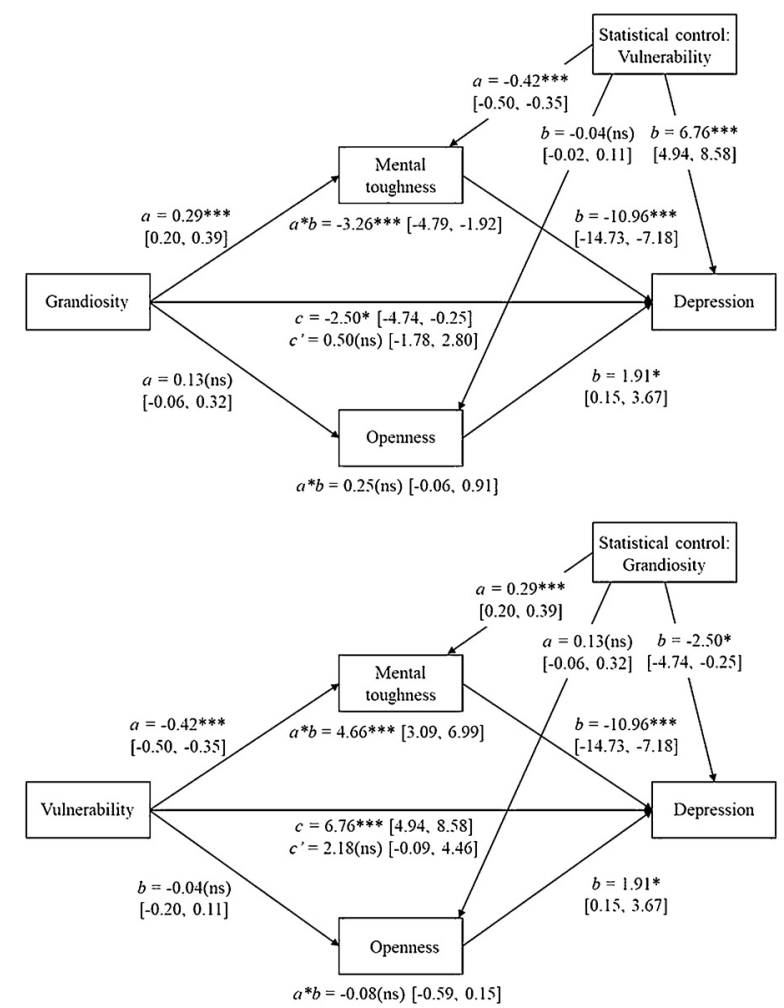

Fig. 3. Models 3 and 4: Mediation effects of Mental Toughness and openness in the relation between grandiosity, vulnerability and depression. Note. $\mathrm{R}^{2}$ for Model $3=44.75 \%$, $R^{2}$ for Model $4=44.75 \%$; results are based on 1000 bootstrap samples: values in square brackets indicate bias-corrected $95 \%$ confidence intervals; ${ }^{*} p<$ $0.05,{ }^{* * *} p<0.001$

for $\mathrm{GN}$ and $\mathrm{VN}$ ) reported a non-significant total effect, $c=0.79,95 \%$ CI $[-1.10,2.70]$.

Analysis of MT subfactors for Model 3 revealed significant indirect effects of GN on DS through Control, $a^{*} b=-1.38,95 \% \mathrm{CI}$ $[-2.87,-0.33]$, and Confidence, $a^{*} b=-1.53,95 \% \mathrm{CI}[-3.33,-0.38]$. For Model 4, significant indirect effects of VN on DS occurred via Control, $a^{*} b=2.62,95 \% \mathrm{CI}[0.39,-5.40]$, and Confidence, $a^{*} b=1.78$, $95 \%$ CI $[0.44,3.48]$. Similar to Models 3 and 4, OE did not exert a mediating effect

In comparison with Study 1 and 2, Model 5 (Fig. 4) indicated SN positively predicted MT and OE ( $a$ weights). MT evinced a negative relationship with DS, and OE positively predicted DS ( $b$ weights). MT had a negative mediating effect, whereas OE exhibited a positive mediating effect ( $a^{*} b$ weights). Weaker mediation effects were evident in the context of reversed relationships for both MT $\left(a^{*} b\right.$ weight $=-0.02$ vs. -4.58$)$ and $\mathrm{OE}\left(a^{*} b\right.$ weight $=0.01$ vs. 0.47$)$.

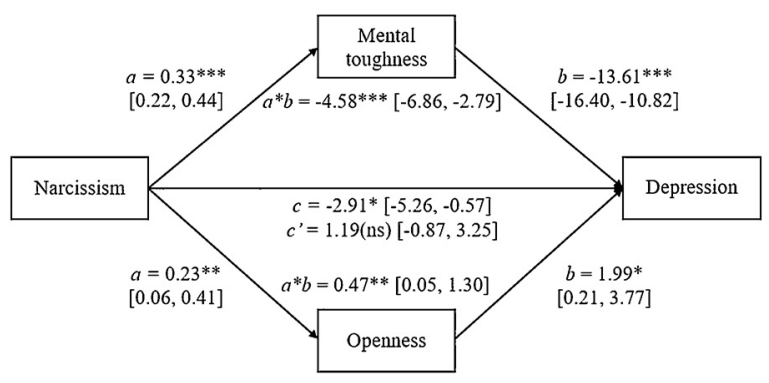

Fig. 4. Model 5: Mediation effects of Mental Toughness and openness in the relation between narcissism and depression. Note. $\mathrm{R}^{2}$ for final model $=43.40 \%$; results are based on 1000 bootstrap samples; values in square brackets indicate bias-corrected $95 \%$ confidence intervals; ${ }^{*} p<0.05,{ }^{* *} p<0.01,{ }^{* * *} p<0.001$. 
Assessing SN as mediator of MT-DS (controlling for OE) revealed a non-significant indirect effect, $a^{*} b=0.68,95 \% \mathrm{CI}[-0.39,2.08]$.

Lastly, given $\mathrm{OE}$ demonstrated a non-significant correlation with DS but a significant mediating effect, controlling for MT and SN revealed a significant association, partial $r=0.19, p=0.028$. This indicates that MT and SN inflated the link between OE and DS. The results of these further analyses are consistent with Study 1 and Study 2. Data for Study 3 available through figshare: https://doi. org/10.6084/m9.figshare.6887135.

\section{Discussion}

The path model, from Subclinical Narcissism (SN) to higher Mental Toughness (MT) to a positive outcome, is a reliable and strong predictor of lower symptoms of depression (DS). Explicitly, the results demonstrate that SN, assessed with the SD3 [28], increases MT resulting in significantly lower DS in three independent samples. Importantly, the results were stable across studies explaining almost $30 \%$ of the variation in DS. Assessment of MT subfactors replicated these results in relation to Control and Confidence. Considering the conceptual link between MT Challenge and the Big Five trait of Openness to Experience (OE), the authors extended the proposed mediation model showing (in Study 2) that SN may decrease DS through $\mathrm{OE}$. Study 3, however, did not replicate this finding. This was possibly due to small sample size.

Previous investigations $[15,16]$ have acknowledged that, scores for SN obtained using the SD3 might be biased towards assessing narcissism as a prosocial trait, linked to healthy self-esteem, rather than evaluating the antisocial aspects of narcissism (see also [43]). To account for this bias, the researchers included an additional valid measure of SN, the short form of the FFNI [29], which differentiates between Grandiose SN and Vulnerable SN. This illustrated that GN may predict lower psychiatric symptoms (DS) through MT. Whereas VN exerted a positive indirect effect on DS through MT explaining more than $40 \%$ of the variation in DS.

This investigation shares well-reported limitations with other research in the domains of personality and psychopathology. Namely, self-report data may be influenced by common-method variance [44], and social desirability, particularly in the context of the assessment of a "dark" trait (narcissism). Another potential limitation of this investigation is that the researchers did not employ survey validity checks [45] to ensure that measures were reliably completed. This does not appear to have significantly influenced the results because Study 3 (completed via pen and paper) produced similar findings (correlations and mediation models) to Study 1 and 2.

Additionally, the studies were cross-sectional, which precludes definitive conclusions concerning the causal order of the variables. Mitigating this concern, a test of reverse relationships supported the hypothesised variable order, and previous longitudinal work is consistent with the notion that Mental Toughness mediates the effects of narcissism (cf. [15]).

The present investigation provides new and counterintuitive insights into the role of a seemingly "dark" trait in reducing indirectly and significantly psychiatric symptoms in the general population. Current (and previous) findings, suggest that simply including the SN into the DT, as a trait that links to poor and toxic psychosocial outcomes, requires revision. This suggestion finds partial support in a recent large meta-analysis and critical review of the literature on the DT traits: the study failed to report statistically significant correlations between $\mathrm{SN}$ and various measures of negative psychosocial outcome, such as, antisocial tactics, aggression, sex-related issues and morality problems (with the exception of a weak positive correlation between SN and interpersonal difficulties), when controlling for SP and Machiavellianism [46].

\section{Conclusion}

The present investigation has direct theoretical and indirect applied implications. The findings support the view that SN is a complex personality trait involving both positive (grandiose) and negative (vulnerable) aspects. Exploring its relation to prosocial traits, such as MT, can be particularly helpful when trying to identify and promote SN's adaptive tendencies. Studying the proposed path model from SN to higher MT, while considering other personality traits (e.g. Openness to Experience) and the distinction between GN and VN, may explain and predict variation in psychiatric symptoms. Considering the malleability of personality traits, joint intervention programmes could promote the adaptive-rather than maladaptive-aspects of SN and train MT in an attempt to reduce DS and possibly other psychiatric symptoms.

\section{Data deposition}

Data is accessible through figshare:

https://doi.org/10.6084/m9.figshare.6887123(Study1); https:// doi.org/10.6084/m9.figshare.6887132(Study2); https://doi.org/ 10.6084/m9.figshare.6887135(Study3).

\section{References}

[1] Kotov R., Gamez W, Schmidt F, Watson D. Linking "big” personality traits to anxiety, depressive, and substance use disorders: A meta-analysis. Psychol Bull 2010;136(5):768-821.

[2] Widiger T. Personality and psychopathology. World Psychiatry 2011;10 (2):103-6.

[3] Ferrari A, Charlson F, Norman R, Patten S, Freedman G, Murray C, et al. Burden of Depressive Disorders by Country, Sex, Age, and Year: Findings from the Global Burden of Disease Study 2010. PLoS Med 2013;10(11)e1001547.

[4] Cuijpers P, Vogelzangs N, Twisk J, Kleiboer A, Li J, Penninx B. Comprehensive meta-analysis of excess mortality in depression in the general community versus patients with specific illnesses. Am J Psychiatry 2014;171(4):453-62.

[5] Paulhus D, Williams K. The dark triad of personality: narcissism, machiavellianism, and psychopathy. J Res Pers 2002;36(6):556-63.

[6] Miller J, Hoffman B, Gaughan E, Gentile B, Maples J, Keith Campbell W. Grandiose and vulnerable narcissism: a nomological network analysis. J Pers 2011;79(5):1013-42.

[7] Dickinson K, Pincus A. Interpersonal analysis of grandiose and vulnerable narcissism. J Pers Disord 2003;17(3):188-207.

[8] Vize C, Lynam D, Collison K, Miller J. Differences among dark triad components: a meta-analytic investigation. Personal Disord.: Theory, Res Treat 2018;9(2):101-11.

[9] Besser A, Zeigler-Hill V. Pathological forms of narcissism and perceived stress during the transition to the university: the mediating role of humor styles. IJSM 2011;18(3):197-221.

[10] Annen H, Nakkas C, Sadeghi Bahmani D, Gerber M, Holsboer-Trachsler E, Brand S. Vulnerable narcissism as key link between dark triad traits, mental toughness, sleep quality and stress. Eur Psychiatry 2017;41:S261.

[11] Veselka L, Schermer J, Vernon P. The Dark Triad and an expanded framework of personality. Personal Individ Differ 2012;53(4):417-25.

[12] Sedikides C, Rudich E, Gregg A, Kumashiro M, Rusbult C. Are normal narcissists psychologically healthy?: self-esteem matters. J Pers Soc Psychol 2004;87 (3):400-16

[13] Onley M, Veselka L, Schermer J, Vernon P. Survival of the scheming: a genetically informed link between the dark triad and mental toughness. Twin Res Hum Genet 2013;16(06):1087-95.

[14] Sabouri S, Gerber M, Bahmani DS, Lemola S, Clough PJ, Kalak N, et al Examining Dark Triad traits in relation to mental toughness and physical activity in young adults. Neuropsychiatr Dis Treat 2016;12:229.

[15] Papageorgiou K, Wong B, Clough P. Beyond good and evil: exploring the mediating role of mental toughness on the Dark Triad of personality traits. Personal Individ Differ 2017;119:19-23.

[16] Papageorgiou K, Malanchini M, Denovan A, Clough P, Shakeshaft N, Schofield K, et al. Longitudinal associations between narcissism, mental toughness and school achievement. Personal Individ Differ 2018:131:105-10.

[17] St Clair-Thompson H, Bugler M, Robinson J, Clough P, McGeown S, Perry J. Mental toughness in education: exploring relationships with attainment, attendance, behaviour and peer relationships. Educ Psychol 2014;35(7):886-907.

[18] Lin Y, Mutz J, Clough P, Papageorgiou K. Mental Toughness and Individual Differences in Learning, Educational and Work Performance, Psychological Well-being, and Personality: A Systematic Review. Front Psychol 2017;11 (8):1345.

[19] Gerber M, Brand S, Feldmeth A, Lang C, Elliot C, Holsboer-Trachsler E, et al Adolescents with high mental toughness adapt better to perceived stress: a 
longitudinal study with Swiss vocational students. Personal Individ Differ 2013;54(7):808-14.

[20] Gerber M, Kalak N, Lemola S, Clough P, Perry J, Pühse U, et al. Are Adolescents With High Mental Toughness Levels More Resilient Against Stress? Stress Health 2012;29(2):164-71.

[21] Mutz J, Clough P, Papageorgiou K. Do Individual Differences in Emotion Regulation Mediate the Relationship Between Mental Toughness and Symptoms of Depression? J Individ Differ 2017;38(2):71-82.

[22] Furnham A, Hughes D, Marshall E. Creativity, OCD, narcissism and the big five. Think. Skills Creativity 2013;10:91-8.

[23] Horsburgh V, Schermer J, Veselka L, Vernon P. A behavioural genetic study of mental toughness and personality. Personal Individ Differ 2009;46(2):100-5.

[24] Clough P, Earle K, Sewell D. Mental toughness: the concept and its measurement. In: Cockerill IM, editor. Solutions in sport psychology. Boston, MA: Cengage Learning; 2002. p. 32-43.

[25] Bajorski P. Statistics for imaging, optics, and photonics. Wiley series in probability and statistics. 2011.

[26] Montgomery D, Peck E, Vining G. Introduction to linear regression analysis. $3^{\text {rd }}$ ed. New York, NY: John Wiley \& Sons; 2011.

[27] Byrne B. Structural equation modeling with Amos: basic concepts, applications, and programming. 3rd ed. New York, NY: Routledge; 2016.

[28] Jones D, Paulhus D. Introducing the short dark triad (SD3). Assessment 2013;21 (1):28-41.
[29] Sherman E, Miller J, Few L, Campbell W, Widiger T, Crego C, et al. Development of a short form of the five-factor narcissism inventory: the FFNI-SF. Psychol Assess 2015;27(3):1110-6.

[30] John OP, Srivastava S. The big five trait taxonomy: history, measurement, and theoretical perspectives. Handb Personal.: Theory Res 1999;2:102-38.

[31] Kroenke K, Spitzer R, Williams J. The PHQ-9. J Gen Intern Med 2001;16 (9):606-13.

[32] Beck A. An inventory for measuring depression. Arch Gen Psychiatry 1961;4 (6):561.

[34] Perry J, Clough P, Crust L, Earle K, Nicholls A. Factorial validity of the mental toughness Questionnaire-48. Personal Individ Differ 2013;54(5):587-92.

[42] Hayes Bolin J, Andrew F. Introduction to mediation, moderation, and conditional process analysis: a regression-based approach. New York, NY: The Guilford Press. JEM; 2014. p. 335-7 51(3).

[43] Maples J, Lamkin J, Miller J. A test of two brief measures of the dark triad: the dirty dozen and short dark triad. Psychol Assess 2014;26(1):326-31.

[44] Podsakoff P, MacKenzie S, Lee J, Podsakoff N. Common method biases in behavioral research: a critical review of the literature and recommended remedies. J Appl Psychol 2003;88(5):879-903.

[45] Becker SP, Dvorsky MR, Holdaway AS, Luebbe AM. Sleep problems and suicidal behaviors in college students. J Psychiatr Res 2018;99:122-8.

[46] Muris P, Merckelbach H, Otgaar H, Meijer E. The malevolent side of human nature. Perspect Psychol Sci 2017;12(2):183-204. 